\title{
SURFACE-MICROMACHINED 1MHZ OSCILLATOR WITH LOW-NOISE PIERCE CONFIGURATION
}

\author{
Trey A. Roessig, Roger T. Howe, and Albert P. Pisano \\ University of California, Berkeley \\ Berkeley, CA 94720
}

James H. Smith

Sandia National Laboratories

Albuquerque, NM 87185

\begin{abstract}
A prototype high-frequency tuning fork oscillator has been fabricated and tested in an integrated surface micromachining technology. The amplifier circuitry uses a capacitive current detection method, which offers superior noise performance over previous resistive methods. The prototype device has an output frequency of $1.022 \mathrm{MHz}$ and exhibits a noise floor of $-88 \mathrm{dBc} / \mathrm{Hz}$ at a distance of $500 \mathrm{~Hz}$ from the carrier. The dominant source of frequency instability is the nonlinearity introduced by the use of parallel-plate actuation.
\end{abstract}

\section{INTRODUCTION}

Oscillators appear in a wide variety of modern applications. They are used extensively in communication systems, both as frequency references and voltage-controlled oscillators. With the current trend towards smaller and more integrated systems, these applications would benefit greatly from the ability to integrate lownoise micromachined oscillators or arrays of oscillators onto the chip [1]. Oscillators are also used in number of sensors, including resonant sensors [2] and vibratory rate gyroscopes [3]. An improvement in the oscillator noise in one of these sensors results in an improvement in the noise floor of the sensor itself.

Previous surface-micromachined oscillators [2-5] have used resistive methods to detect motional current, introducing a relatively high noise floor into the system. This noise floor, in addition to causing its own phase noise far from the output frequency, forces the mechanical structure to vibrate at a high amplitude so the carrier can be seen. This causes nonlinearity in the vibration, which has been shown to be the dominant source of phase noise near the carrier [6].

This paper describes a prototype surface-micromachined oscillator based on a capacitive current detection method. Because
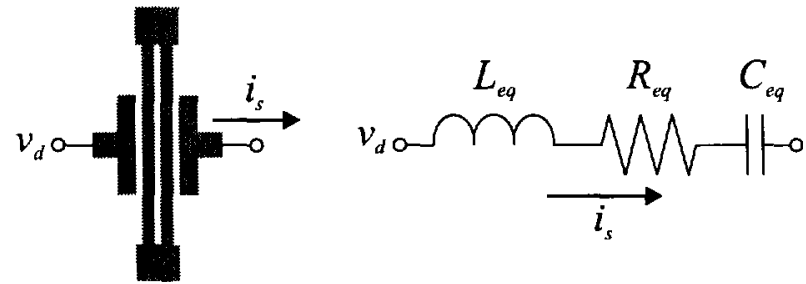

Figure 1. Electrical behavior of micromachined tuning fork. The motion of the tines translates to current on the output node.

of the large impedance at the sense node, the capacitive method introduces smaller amounts of input-referred current noise than the resistive method. This method of detection integrates the current at the input node, so it requires a second integration to properly complete the oscillation loop. This double integration loop is known in the oscillator literature as the Pierce configuration [7].

This paper compares the various analog current-sensing methods and demonstrates that for low-noise applications, the capacitive method is superior. Next, the implementation of the oscillation amplifier is presented. Following this, the mechanical structure and electrostatic actuation are discussed. Finally, the experimental results from the system are presented.

\section{CURRENT DETECTION}

Surface-micromachined resonators, like quartz resonators, can be modeled as a series LCR circuit, as shown in Figure 1 [8]. A voltage is applied to the drive electrode of the resonator, and the response is a motional current on the sense node. So the problem of detecting resonator motion reduces to one of detecting current.

In CMOS technology, there are only a few ways of detecting
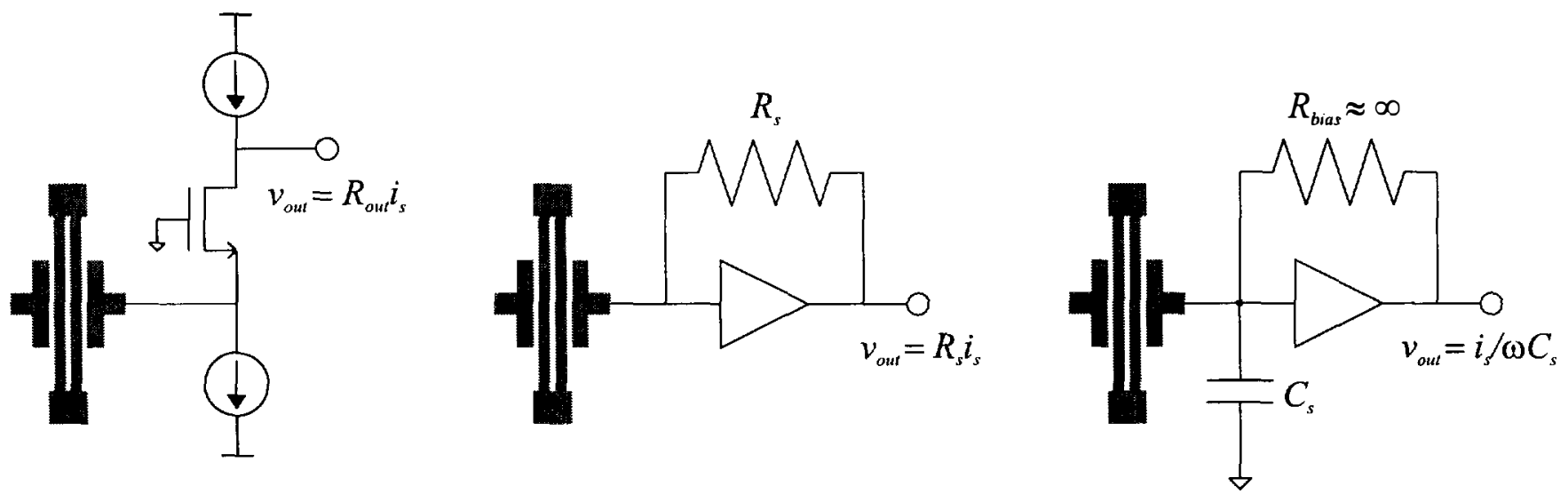

Figure 2. Three potential methods of sensing motional current. From left to right, they are: common base detection, resistive detection, and capacitive detection. Each method introduces an equivalent current noise at the input node to the circuit. 


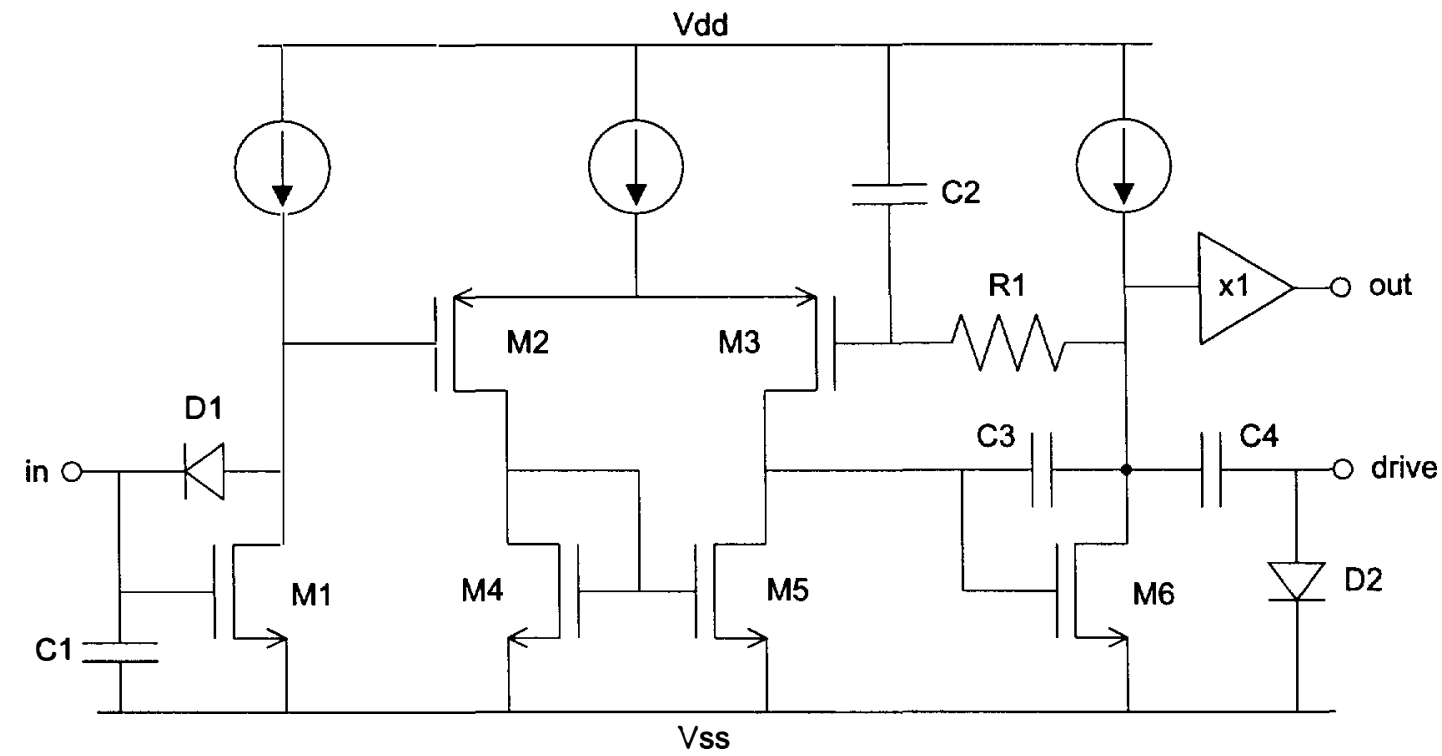

Figure 3. Simplified schematic of Pierce amplifier implementation. Motional current is integrated at the input node. The M2-M3 differential pair serves as the input to the two-stage operational amplifier used both in the bias loop and as the second integration. The RIC2 pair serves as the bias pole, and the compensation capacitor C3 serves as the integration pole. The output signal is AC-coupled to the resonator through C4-D2.

current using non-sampled techniques. Three of these methods are shown in Figure 2. The first of these is the common base configuration, but the analysis holds for any other feedback configuration where the sense electrode is directly connected to the source or drain of a MOS transistor. In these cases, assuming the transistor is in saturation, the current noise introduced by the MOS is:

$$
\bar{i}_{n}^{2}=4 k T\left(\frac{2}{3} g_{m}\right)
$$

where $i_{n}{ }^{2}$ is the equivalent current on the sense node, and $g_{m}$ is the transconductance of the transistor. Note that this equation does not include the effects of the bias and load current sources, which add additional noise.

The second method shown in Figure 2 is the resistive method. A resistor in series with the resonator can be used to create a voltage proportional to the motional current. This voltage can then be measured with a MOS gate without directly adding to the current noise on the node. With this detection method, the current noise at the sense node (ignoring the equivalent input noise of the amplifier) is:

$$
\vec{i}_{n}^{2}=\frac{4 k T}{R_{s}}
$$

The third method shown in Figure 2 is the capacitive detection method. This method involves integrating the output current by putting a capacitor in series with the resonator and measuring the resulting voltage. A resistor is still required for biasing purposes, but is large enough that the impedance at the sense node is dominated by the capacitor. In this method, the input-referred current noise is simply:

$$
\bar{i}_{n}^{2}=\frac{4 k T}{R_{\text {bias }}}
$$

It can be seen, then, that the lowest noise is achieved with the capacitive method. In the source/drain case, the $g_{m}$ of the input transistor would have to be very small to lower the noise to a level comparable to the other methods. Unless the MOS is biased with an extremely small current, it is difficult to get a low enough $g_{m}$ to do this. The resistive method is better, but as the sense resistor is increased to reduce the noise, the bandwidth of the input stage becomes unacceptably small. The capacitive pickoff method deliberately operates past the bandwidth of the input stage, so that it will integrate the current. This allows $R_{\text {bias }}$ to be arbitrarily large, giving the capacitive method the lowest noise of the three.

Operating past the RC constant also has advantages in terms of resonator loading. The impedance at the input node for a given frequency can be shown to be [7]:

$$
Z_{\text {in }}=\frac{R_{\text {bias }}}{R_{\text {bias }}^{2} C_{s}^{2} \omega^{2}+1}-j \frac{R_{\text {bias }}^{2} C_{s} \omega}{R_{\text {bias }}^{2} C_{s}^{2} \omega^{2}+1}
$$

where $\omega$ is the frequency in question.

Maintaining a high Q (low energy loss) in the system is important in sustaining oscillation and reducing phase noise [8]. Resistive impedances in series with the structure increase the damping and reduce the $\mathbf{Q}$ of the resonator. Reactive impedances change the frequency of the resonator itself, but have no effect on

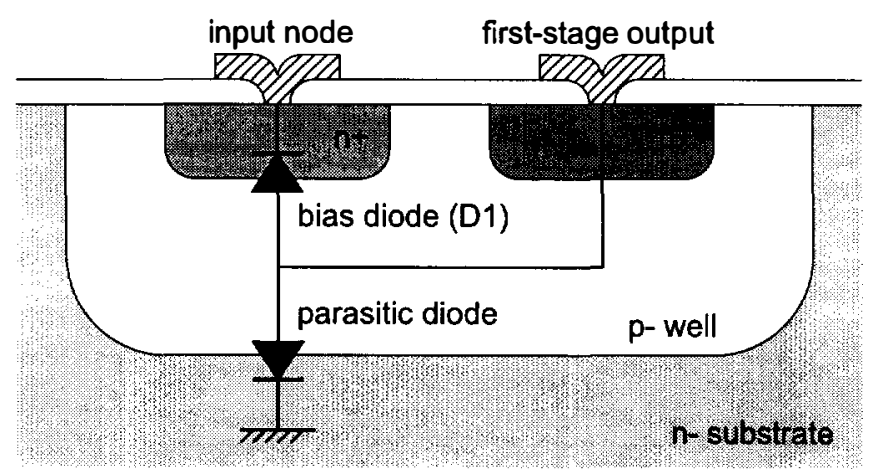

Figure 4. Illustration of the parasitic diode. Layout is arranged so that the parasitic diode is on the lower-impedance output node. 


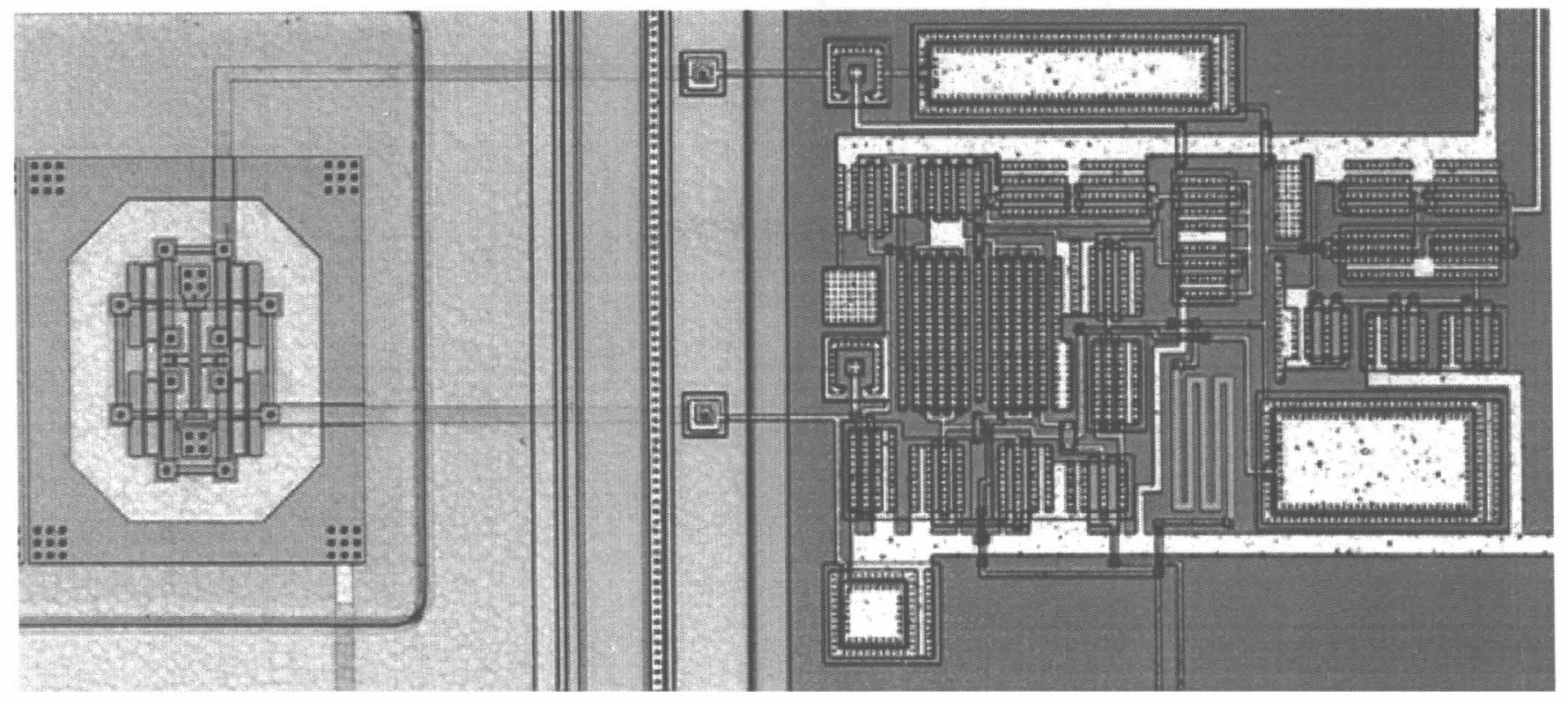

Figure 5. Die photo of fabricated oscillator. The resonator structure is fabricated in a bulk-etched well before CMOS processing. The die area shown here is approximately $450 \mu \mathrm{m}$ by $750 \mu \mathrm{m}$.

the Q. Since the oscillator is designed to operate at a frequency well above $\left(R_{\text {bias }} C_{s}\right)^{-1}$, Equation 4 shows that the impedance at the input node is primarily reactive. This implies that, despite the presence of a large DC resistance, the input impedance does not reduce the $\mathrm{Q}$ of the resonator appreciably. What it will do is change the resonator frequency slightly. Fortunately, because of the stiffness of surface micromachined structures, this frequency pulling effect is minor [9].

\section{AMPLIFIER IMPLEMENTATION}

While the arbitrarily large resistors described above result in good noise performance for the amplifier, they can be difficult to realize. A few possible solutions are subthreshold MOS devices [3] or zero-biased diodes [10]. The latter is used here.

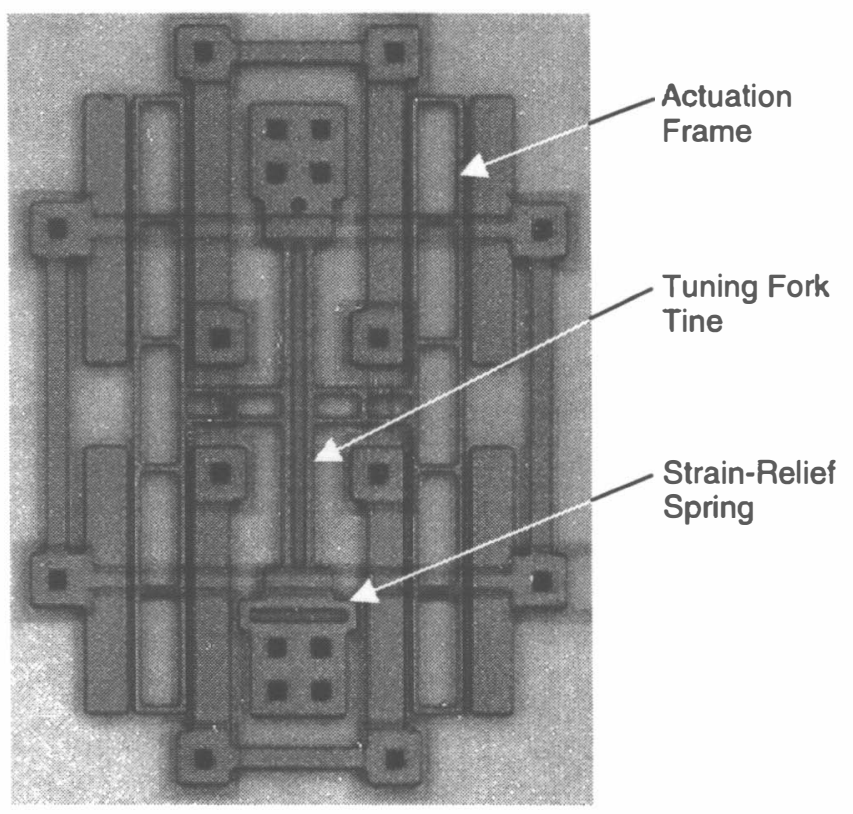

Figure 6. Close-up view of the resonator structure. The tines are $2 \mu \mathrm{m}$ wide, $60 \mu \mathrm{m}$ long, and $2 \mu \mathrm{m}$ thick.
Another issue with the Pierce implementation is the requirement of a second integration. It is difficult to get a pole low enough to be sufficient with a simple RC filter. In addition, because of the high impedance input node, small leakage currents can have a large effect on the bias point. The second stage must not only integrate the signal, but must be able to bias correctly for a large range of input voltages.

The circuit implementation chosen for the prototype device is shown in Figure 3. The first stage is a single common-source stage biased by a diode placed across the drain and gate. Since there is no DC current flow across the diode, ignoring leakage currents, the bias voltage across the diode is near zero. This results in a high small-signal impedance. A capacitor on the input node guarantees that the front end of the circuit is an integrator at the frequency of interest. It also reduces the effect of the parasitic feedback capacitance across the diode.

The layout of the bias diode (D1 in Figure 3) is important. The diode is placed in a p-type well, as shown in Figure 4. The device is laid out so that the parasitic weli-substrate diode does not shunt the high-impedance input node. This prevents the leakage currents from the parasitic diode from affecting the input bias of the amplifier, which could render the circuit inoperable. This arrangement also removes the well depletion capacitance from the input node.

The remainder of the circuit is a simple two-stage op-amp with a Miller-compensated pole. The bias network around the amplifier forces the DC bias to be whatever the output voltage of the first stage is. In this case, the pole of the bias network was set with an off-chip capacitor. The bias network has no effect at the oscillation frequency, so the amplifier runs open-loop. The Miller pole serves as the second integration, completing the loop with the correct phase necessary for oscillation.

\section{RESONATOR STRUCTURE}

The resonator structure is a simple double-ended tuning fork. The reason for the use of this structure is the availability of high frequencies while maintaining a reasonable aspect ratio for the beams. One disadvantage of this structure, however, is its sensitivity to axial strains. This drawback has been reduced 


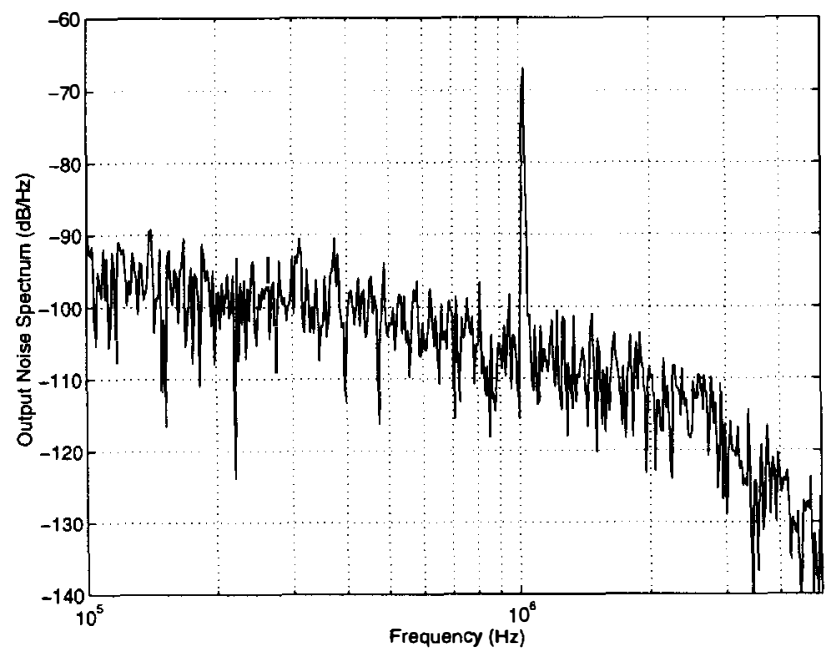

Figure 7. Far-field output noise spectrum of integrated oscillator. No higher harmonics of the oscillation are visible.

somewhat by placing a strain relief spring at one end of the tuning fork.

The tuning fork tines are actuated with a balanced parallel plate structure. Each tine has equal sets of drive and sense plates, and the two tines are wired in parallel. This rejects the symmetric vibration mode of the structure, exciting only the high-Q antisymmetric mode.

The choice of parallel-plate actuation was made to lower the series resistance of the structure, but comes at a price. Parallelplate actuators provide more transduction and add less mass to the structure than their comb-drive counterparts. This greatly reduces the series resistance of the structure, making it easier to sustain oscillation. The drawback to these actuators, however, is the nonlinearity associated with them. The force-displacement curve for the movable portion has a non-zero slope at the center, implying that the electrostatic forces impart a "negative electrical spring" to the structure [3]. This directly changes its natural frequency. Since the frequency change is a function of the DC voltage across the plates, the frequency of the oscillator becomes a function of that voltage. This implies the increased transduction has come at the price of reduced oscillator stability.

The structure itself is made of $2 \mu$ m-thick polysilicon and consists of two parallel tines, each $2 \mu \mathrm{m}$ wide by $60 \mu \mathrm{m}$ long. Each actuation frame has stiffening bars to keep it from developing its own parasitic oscillation. All area underneath the structure that is not used for interconnect is covered with a polysilicon shield and shorted to the resonator to reduce feedthrough currents and eliminate pulldown of the structure itself.

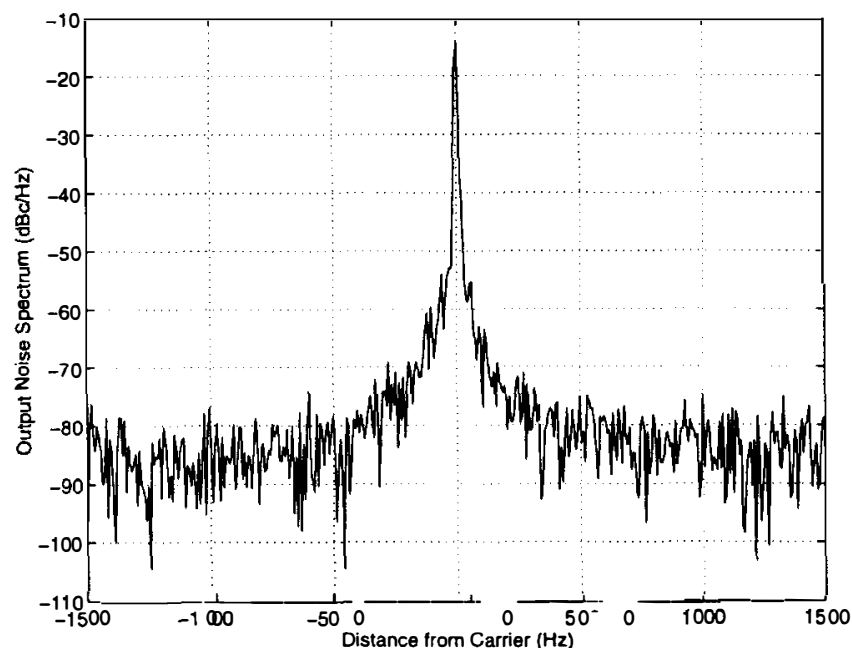

Figure 8. Near-carrier output noise spectrum of oscillator. The sidebands fall behind the noise floor at $500 \mathrm{~Hz}$ from the carrier.

The prototype oscillator described above was fabricated in Sandia National Lab's embedded MEMS/CMOS surface micromachining process [11]. A die photo of the resonator and oscillator are shown in Figure 5, and a close-up of the structure itself is shown in Figure 6.

\section{EXPERIMENTAL RESULTS}

The amplifier behavior can be tested by applying an input signal on the structural node of the resonator while the chip is held at atmospheric pressure. The amplifier input node sees a voltage capacitively divided between the resonator static capacitance and the input capacitor. This signal can be used to characterize the amplifier while the ambient pressure suppresses the oscillation. The behavior of the circuit is in line with expectations, with the integration pole appearing at about $350 \mathrm{kHz}$.

Mechanical oscillation is sustained when the chip is held in vacuum. The nominal output frequency of the device is 1.022 $\mathrm{MHz}$. The far-field output spectrum from the oscillator is shown in Figure 7, as taken by a HP 4195A Spectrum Analyzer. Note the integrating nature of the circuit can be inferred from the downward-sloping noise. The $350 \mathrm{kHz}$ pole can be seen in the noise shape, along with the $3 \mathrm{MHz}$ bandwidth of the buffer used to drive the analyzer. There are no other peaks visible, implying the second and third harmonics are at least $45 \mathrm{~dB}$ below the carrier.

Oscillation is sustained for a surprisingly narrow range of structural polarization voltages, between $6.5 \mathrm{~V}$ and $7.5 \mathrm{~V}$. For polarization voltages less than that, no oscillation begins, implying a large series resistance in the structure. For voltages higher than

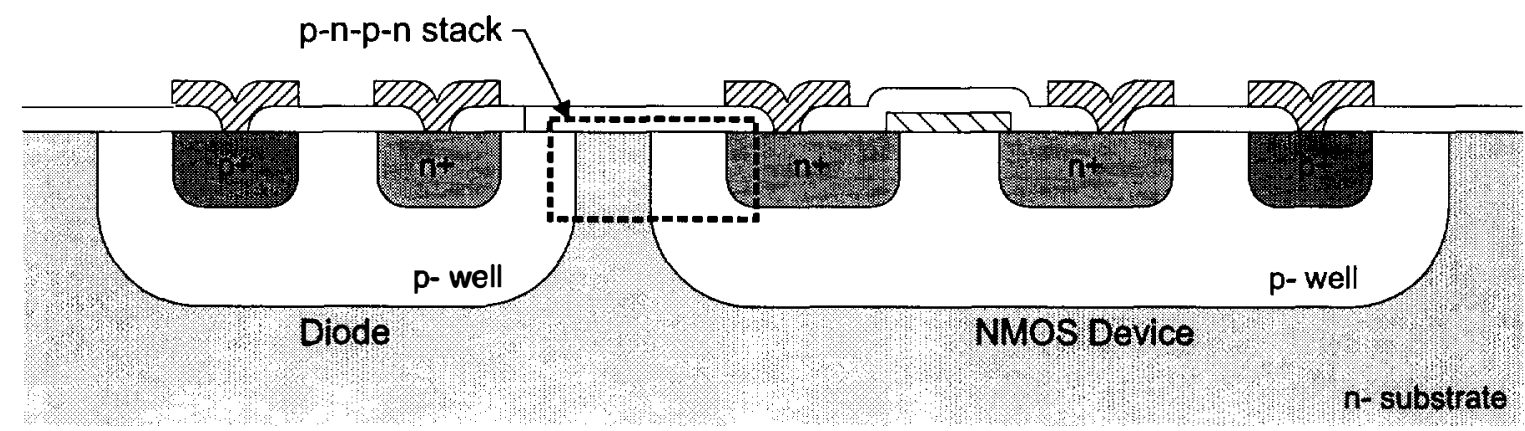

Figure 9. Illustration of the pnpn stack that causes latchup in the front end of the circuit. Future layouts will include substrate contacts between the input devices and the bias diode. 


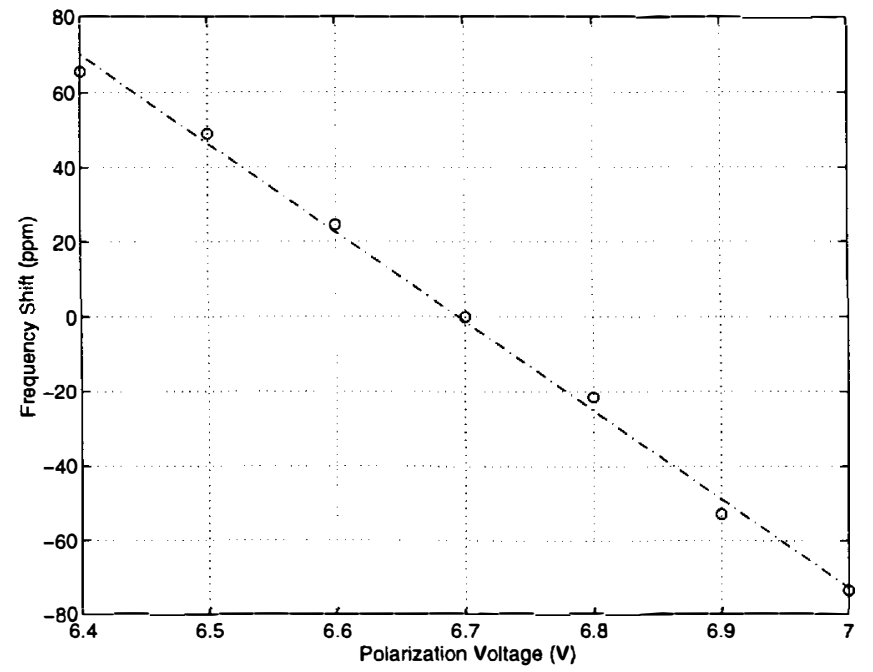

Figure 10. Polarization voltage effect on natural frequency. The downward trend and linearity imply a direct effect on the natural frequency.

$7 \mathrm{~V}$, a large distortion can be seen in the waveform. For voltages higher than $7.5 \mathrm{~V}$, the input stage of the amplifier latches up. The layout of the bias diode next to the input NMOS, a cross-section of which is shown in Figure 9, creates a pnpn layer stack which results in latchup for large signals [12]. While a substrate contact was placed near the diode, it apparently was not enough to prevent this phenomenon. Future designs will include substrate contacts between the diode and input transistor.

The near-carrier output spectrum is shown in Figure 8. The noise floor of the circuit near the carrier is $-106 \mathrm{~dB} / \mathrm{Hz}$ and the carrier power varies between -18 and $-29 \mathrm{~dB}$, depending on the polarization voltage in use. This results in a best-case noise floor of $-88 \mathrm{dBc} / \mathrm{Hz}$ at a distance of $500 \mathrm{~Hz}$ from the carrier. This number would have dropped even further, if not for limitation on the output power caused by the latchup problem. Nevertheless, this is more than a $20 \mathrm{dBc} / \mathrm{Hz}$ improvement over previous surfacemicromachined tuning fork oscillators [2].

The oscillator does not suffer from the mechanical stiffening nonlinearity seen in previous tuning fork structures [6], or at least it is too small to be detectable. The dominant source of voltagefrequency dependence present is that of the electrostatic nonlinearity introduced by the parallel-plate actuation. Figure 10 shows the variation in frequency with changes in the polarization voltage. This plot shows a linear downward slope, indicative of direct natural frequency shifts. The slope of the curve, $250 \mathrm{~Hz} / \mathrm{V}$, is in good agreement with the model prediction of the nonlinearity.

\section{CONCLUSIONS}

A prototype high-frequency surface-micromachined oscillator has been fabricated and tested. Despite some difficulty with latchup phenomena in the input stage, the oscillator is still a large improvement over previous implementations from a background noise perspective. The dominant source of frequency shifts is the electrostatic nonlinearity present in the actuation. Future designs will incorporate extra protection against latchup, and will minimize electrostatic effects on natural frequency.

\section{ACKNOWLEDGEMENTS}

The authors would like to acknowledge the students of the Berkeley Sensor \& Actuator Center for their help and support,
Sandia National Labs for fabrication of the oscillators, and the DARPA MEMS program for financial support of this research.

Sandia is a multi-program laboratory operated by the Sandia Corporation, a Lockheed-Martin company, for the US Department of Energy under contract DE-AC04-94AL85000.

\section{REFERENCES}

1. C. T.-C. Nguyen, "Microelectromechanical devices for wireless communications", Eleventh IEEE International Workshop on Microelectromechanical Systems (MEMS '98), Heidelberg, Germany, 1998, pp. 1-7

2. T. A. Roessig, R. T. Howe, A. P. Pisano, and J. H. Smith, "Surface-micromachined resonant accelerometer", Ninth International Conference on Solid-State Sensors and Actuators (Transducers '97), Chicago, IL, 1997, pp. 859-862

3. W. A. Clark, R. T. Howe, and R. Horowitz, "Surfacemicromachined vibratory rate gyroscope", Solid State Sensor and Actuator Workshop, Hilton Head, SC, 1996, pp. 283-287

4. C. T.-C. Nguyen and R. T. Howe, "CMOS micromechanical resonator oscillator", IEEE International Electron Devices Meeting, Washington, DC, 1993, pp. 127-134

5. T. A. Roessig, A. P. Pisano, and R. T. Howe, "Surface micromachined resonant force sensor", Proceedings of the ASME Dynamic Systems and Control Division, DSC-Vol. 57-2, 1995 ASME IMECE, San Francisco, CA, 1995, pp. 871-876

6. T. A. Roessig, R. T. Howe, A. P. Pisano, and J. H. Smith, "Nonlinear mixing in surface micromachined tuning fork oscillators", IEEE Frequency Control Symposium, Orlando FL, 1997, pp. 778-782

7. Robins, W. P., Phase Noise in Signal Sources, London, Peter Peregrinus, Ltd., 1982

8. C. T.-C. Nguyen, "Micromechanical signal processors", $\mathrm{Ph} . \mathrm{D}$. Dissertation, UC Berkeley, 1994

9. T. A. Roessig, "Integrated MEMS tuning fork oscillators for sensor applications", Ph.D. Dissertation, UC Berkeley, 1998

10. G. K. Fedder and R. T. Howe, "Multimode digital control of a suspended polysilicon microstructure", Joumal of Microelectromechanical Systems, Vol. 5, No.4, 1996, pp. 283-297

11. J. H. Smith, et al., "Embedded Micromechanical Devices for the Monolithic Integration of MEMS and CMOS", IEEE International Solid-State Circuits Conference, San Francisco, CA, 1995, pp. 609-612

12. P. R. Gray and R. G. Meyer, Analysis and Design of Analog Integrated Circuits, $3^{\text {rd }}$ Ed., New York, John Wiley \& Sons, Inc., 1993 